\title{
Theodore Millon, una teoría de la personalidad y su patología
}

\author{
Roberto Oscar Sánchezị
}

\begin{abstract}
Resumen
Desde su obra seminal de 1976, Psicopatología moderna: un enfoque biosocial de los aprendizajes erróneos y de los disfuncionalismos, y por más de 30 años Theodore Millon ha desarrollado una ciencia compleja de la personalidad y su patología. En su modelo, el autor postula la existencia de tres polaridades básicas, placer-dolor, activo-pasivo y yootros, para explicar las diferencias entre los diferentes tipos de personalidad. El modelo original de aprendizaje biosocial fue reformulado en 1990 a la luz de los conceptos evolutivos. A lo largo de todo este tiempo, Millon junto a sus seguidores han establecido un paradigma para comprender la personalidad. Al mismo tiempo, há argumentado reiteradamente que una ciencia clínica comprensiva de la personalidad necesita integrar cuatro componentes básicos: teoría o esquemas conceptuales explicativos, una nosología formal, herramientas de evaluación, e intervenciones terapéuticas adecuadas. En este trabajo, se resumen los aspectos primordiales del Modelo de Millon en lo que hace a esos diferentes componentes.
\end{abstract}

Palabras-Clave: Personalidad; Trastornos de la personalidad; Millon; MCMI; MIPS.

\section{Theodore Millon, a theory of the personality and its pathology}

\begin{abstract}
From its seminal work of 1976, Psicopatología moderna: un enfoque biosocial de los aprendizajes erróneos y de los disfuncionalismos, and by more than 30 years Theodore Millon has developed a complex science of the personality and its pathology. In his model, the author postulates the existence of three basic polarities, pleasure-pain, active-passive and I-other, to explain the differences between the different types from personality. The original model of biosocial learning was reformulated in 1990 to the light of the evolutionary concepts. Throughout all this time, Millon next to their followers has established a paradigm to understand the personality. At the same time, it has argued repeatedly that a comprehensive clinical science of the personality needs to integrate four basic components: explanatory theory or conceptual schemes, a formal nosology, suitable tools of evaluation, and therapeutic interventions. In this work, the fundamental aspects of the Model of Millon are transformed in which it does to those different components.
\end{abstract}

Keywords: Personality; Personality disorder; Millon; MCMI; MIPS.

\section{Introducción}

En 1968 la American Psychiatric Association publica la primera revisión del Manual Diagnóstico y Estadístico de los Trastornos Mentales, el DSM-II (APA, 1968), que continuaba a la versión original de 1952. Para entonces, la personalidad y su patología ocupaban un lugar periférico, lejos aún de disponer de un eje diagnóstico propio, situación que tendría lugar recién en la próxima revisión del Manual, el DSM-III, en 1980. En el DSMII los trastornos de la personalidad aparecían en el capítulo V, Trastornos de la personalidad y otros trastornos psíquicos no psicóticos (junto con las desviaciones sexuales, el alcoholismo y las toxicomanías), y se incluían los trastornos paranoide, ciclotímico, esquizoide, explosivo, obsesivo compulsivo, histérico, asténico, antisocial, pasivo agresivo, inadecuado, y la categoría residual de otros tipos.

Poco tiempo después de aparecido el DSM-II, Theodore Millon publica el primer movimiento de una vasta obra donde dejaría sentadas las bases del estudio de la personalidad y su patología en las décadas futuras. En Psicopatologia Moderna. Enfoque biosocial de los aprendizajes erróneos y de los disfuncionalismos (Millon, 1976) Millon propuso una nueva clasificación para los trastornos psiquiátricos, presentando al mismo tiempo una rica y fecunda teoría de los trastornos de la personalidad. La influencia de su modelo fue decisiva para lo que luego sería el Eje II del Manual Diagnóstico y Estadístico de los Trastornos Mentales, a partir de su tercera versión de 1980. Decía en 1976 en la Introducción de su obra:

Lo más representativo del cuerpo central de la psicopatología son los problemas no dramáticos y mundanos de la vida, las ansiedades calladas pero persistentes, las frustraciones repetidas y conflictos inmovilizantes que obstaculizan a millones de americanos día a día. Estos trastornos leves suelen ser considerados como algo que nos "viene dado" como parte del "destino" del hombre o de su "naturaleza". (p. 1) 
En este párrafo introductorio Millon deja sentado un principio que marcaría indeleblemente el estudio de la psicopatología de ahí en más: no sólo los trastornos sintomáticos, más floridos y evidentes, deben formar parte de la nosología sino también los patrones de personalidad patológica, a menudo más larvados y ocultos, de los cuales emergen los trastornos sintomáticos. Así definía el autor esos patrones:

Los patrones de personalidad patológica son características profundamente arraigadas que impregnan todas las facetas de la actividad del individuo (...) Estos patrones derivan de la compleja y secuencial interacción de los factores constitucionales y de la experiencia. Una ver. establecidos en los primeros estadíos de la vida, los patrones patológicos tienden a invadir nuevas esferas y a perpetuarse dentro de círculos viciosos; imponen un modo de vida tan arraigado y automático que el paciente a menudo no advierte su presencia ni sus devastadoras consecuencias. (p. 246)
Otra de las ideas fuertes presentada por el autor en su obra seminal fue que la personalidad patológica puede adquirir diferentes niveles de gravedad. Millon postuló una diferenciación de la gravedad en tres niveles de intensidad: leve, moderada y grave. A medida que la severidad de la patología de la personalidad aumenta, el sabor distintivo de los diferentes estilos de personalidad se atenúa. Como resultado de este proceso el cuadro clínico de individuos con personalidades diferentes se vuelve menos diverso. La figura 1 muestra los prototipos básicos de personalidad de Millon, y el proceso al que se hacía referencia, en forma gráfica. Así, por ejemplo, las personalidades de tipo esquizoide y evitativo derivan en un estilo esquizotípico cuando alcanzan un grado mayor de gravedad.

Como se ve en la Figura 1, el sistema original se conformó con ocho prototipos de personalidad tomados de la nosología psiquiátrica del momento. La innovación estuvo en la manera en que Millon describió y conceptualizó los ocho prototipos en su libro de 1976, apartándose en algunos aspectos de la propuesta del DSM-II.

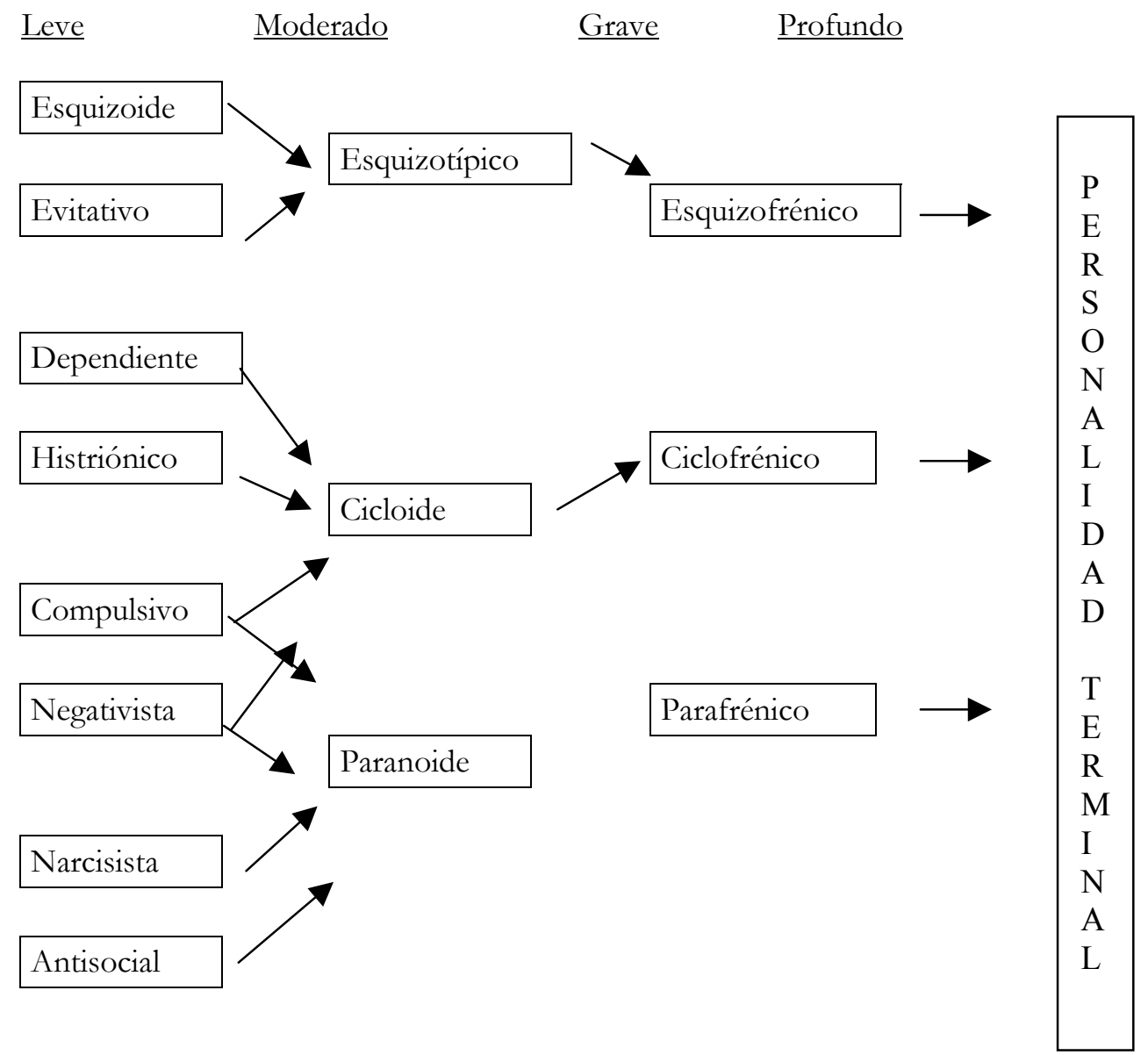

Figura 1 - Modelo original para la descompensación de los prototipos de personalidad en personalidades más severas. Nota: la personalidad esquizoide se llamó asocial en el trabajo original y la personalidad esquizotípica fue llamada esquizoide; estos nombres se cambiaron a los actuales para evitar confusión (Adaptado de Choca, 1999). 
Los prototipos originales de Millon fueron la personalidad asocial luego llamada esquizoide (asténica), ${ }^{2}$ la evasiva (esquizoide), sumisa (inadecuada), gregaria luego llamada histriónica (histérica), narcisista (agregada por Millon, ausente en el DSM-II), agresiva (antisocial), resignada (obsesivo compulsivo), y negativista (explosivo y pasivo).

Las diferencias entre la propuesta de Millon de 1976 y la del DSM-II de 1968 son notorias. En primer lugar, describió versiones menos patológicas de los trastornos de personalidad del DSM-II, creando los patrones de personalidad de severidad leve. En segundo lugar, ajustó sus prototipos de personalidad en un esquema de clasificación lógico y coherente. Este propósito, establecer un sistema de clasificación lógico y coherente, ha estado presente en toda la obra de Millon junto a otros aspectos considerados esenciales para entender la personalidad. Para organizar los ocho prototipos de personalidad, Millon enfocó sobre el tipo de relaciones interpersonales que el individuo establece típicamente y en el modo con que la persona logra su acomodación con el entorno (Choca, 1999).

Los primeros ocho tipos de personalidad se pensaron como estando presentes tanto en personalidades normales como en anormales, mientras que los segundos tres sólo fueron propuestos para personalidades patológicas. Esa primera versión de la teoría estaba dirigida hacia la personalidad normal y la anormal, enlazadas en un continuo. Decía Millon:

\section{La normalidad y la patología son conceptos}

relativos; son cotas arbitrariamente establecidas dentro de un continuum o gradatoria. La psicopatología va modelándose de acuerdo con los mismos procesos y principios que intervienen en el desarrollo y aprendizaje normales. Sin embargo, debido a las diferencias de características especificas, cronología, intensidad o persistencia de determinadas influencias, algunos individuos adquieren hábitos y actitudes caracterizados por una deficiente capacidad de adaptación, mientras que otros no los adquieren. (p. 250)

La diferencia principal, entonces, es que los individuos normales muestran flexibilidad adaptativa en respuesta a su ambiente, mientras que las personas con trastornos de la personalidad exhiben conductas rígidas y desadaptativas. La tendencia a crear círculos viciosos que perpetúan las dificultades y la escasa estabilidad en condiciones de estrés son las otras dos diferencias fundamentales entre las personalidades patológicas y las normales.

Posteriormente, el foco fue puesto en los trastornos de personalidad y los estilos de personalidad normal no fueron descriptos en los textos subsiguientes. Los estilos de personalidad sin trastornos no fueron retomados hasta la publicación del Inventario Millon de Personalidad para Adolescentes (MAPI) y del Inventario Millon de Conductas de Salud (MBHI), ambos de 1982 (Strack, 1999). La propuesta actual de Millon engloba en un mismo marco teórico la personalidad normal y la patológica. El modelo de estilos y dimensiones de la personalidad normal surge del mismo modelo de la personalidad que diferencia y enlaza caracteres sanos y patológicos en un continuo (Millon, 1997a; Millon \& Davis, 1998; Strack, 1999). En la Tabla 1 se presentan los distintos nombres dados por el autor a los diferentes tipos de personalidad de 1976 a la actualidad.

Tabla 1 - Nombres para los estilos de personalidad de 1976 a la actualidad (adaptado de Strack, 1999)

\begin{tabular}{llll}
\hline Psicopatología Moderna & MCMI-II & MAPI - MBHI & MIPS $^{3}$ \\
1976 & 1987 & $1982^{4}$ & 1994 \\
\hline Asocial (pasivo - retraído) & Esquizoide & Introvertido & Retraimiento \\
Evitativo (activo - retraído) & Evitativo & Inhibido & Vacilación \\
Sumiso (pasivo - dependiente) & Dependiente & Cooperador & Concordancia \\
Gregario (activo - dependiente) & Histriónico & Sociable & Comunicatividad \\
Narcisista (pasivo - independiente & Narcisista & Seguro & Firmeza \\
Agresivo (activo - independiente) & Antisocial & Violento & Discrepancia \\
Conformista (pasivo - ambivalente) & Compulsivo & Respetuoso & Conformismo \\
Negativista (activo - ambivalente) & Negativista & Sensitivo & Insatisfacción \\
\hline
\end{tabular}

En diferentes ocasiones, el autor ha argumentado que más que desarrollos independientes de funciones desconectadas, una ciencia madura de la personalidad debe englobar cuatro elementos que trabajan juntos integradamente: teoría, nosología,

2 Entre paréntesis se agrega la denominación correspondiente al DSM-II. evaluación y intervenciones clínicas (Millon, Everly \& Davis, 1995; Millon \& Davis, 1998; Davis, 1999; Millon, 1999a; Millon, 2000; Millon, 2002). La mayoría de las

${ }^{3}$ Inventario Clinico Multiaxial de Millon II (Millon, 1999b).

4 Igual denominación en personalidades normales de "La personalidad y sus trastornos" (Millon \& Everly, 1994).

${ }^{5}$ Inventario Millon de Estilos de Personalidad (Millon, 1997a). 
escuelas de psicoterapia actuales comparten el fracaso al momento de coordinar estos cuatro componentes (Millon, Everly \& Davis, 1995). A continuación se revisan cada uno de estos tópicos de acuerdo a las propuestas de Millon.

\section{Teoría y clasificación}

Una de las críticas más comunes hacia la clasificación de los trastornos de la personalidad que aparece en el DSM-IV (APA, 1995) es la ausencia de un modelo teórico unificado (Widiger, 1999). La propuesta de Millon constituye uno de los aportes más sólidos y de mayor desarrollo para cubrir esa falencia. Su perspectiva teórica adquiere singular importancia en cuanto el autor ha sido un participante influyente en el desarrollo de varias ediciones de la nomenclatura de los trastornos de personalidad.

Durante más de tres décadas, Millon y sus colaboradores desarrollaron y ampliaron la teoría original, produciendo una amplia serie de libros, capítulos de libros y de artículos que reflejan su esfuerzo para construir una ciencia unificada de la personología y la psicopatología (Davis, 1999). Parte de este esfuerzo estuvo dirigido a integrar componentes previamente dispersos dentro de una ciencia clínica integrada (Davis, 1999).

La primera propuesta teórica de Millon estaba basada en un modelo de aprendizaje biosocial (Millon, 1976; Millon \& Everly, 1994) y la noción de refuerzo era el tema unificador del modelo. Al respecto, Millon sintetizaba su propuesta a partir de tres preguntas: ¿qué refuerzos busca el individuo?, ¿dónde procura encontrarlos?, y ¿cómo actúa para poder optar por ellos? El modelo resultante podía ser visualizado como el resultado de tres dimensiones polares que respondían a esas preguntas. Millon decía, entonces, que el refuerzo buscado podía ser la persecución del placer o la evitación de dolor, que ese refuerzo podía ser buscado en sí mismo o en los otros, y finalmente, que el individuo podía actuar de manera activa o de manera pasiva para alcanzarlo. De esta manera, las tres polaridades del modelo original fueron las siguientes: conducta instrumental (como), activo - pasivo; fuente del refuerzo (donde), sí mismo (independiente) - otros (dependiente); naturaleza del refuerzo (que), placer-dolor.

La teoría sostiene que a partir del conocimiento de los defectos de estas tres dimensiones polares es posible derivar de forma deductiva los ocho trastornos de personalidad básicos, combinando la naturaleza (positivo o placer contra negativo o dolor), la fuente (sí mismo versus otros), y las conductas instrumentales (activo contra pasivo). El modelo incluía, además, como se dijo, tres variantes de trastornos de personalidad más severos. Si bien es fácil imaginar ocho modelos de personalidad resultantes del cruce de tres polaridades (como una matriz de $2 \times 2 \times 2$ ) esta no fue la metodología seguida por Millon. Los ocho patrones que derivó son, en cambio, un desbalance o una mezcla desigual de las tres polaridades, lo que según Widiger (1999) constituye un error potencial del modelo. Cuatro patrones se derivan de la naturaleza y fuente de refuerzos: retraído (no busca refuerzos), dependiente (busca refuerzo en los demás), independiente (busca refuerzo en sí mismo), y ambivalente (inseguro respecto a donde buscar refuerzo). Estos cuatro patrones se cruzan con las dos variantes de conducta instrumental (activo, pasivo) para producir ocho modelos de personalidad. Las dos variantes de conducta instrumental representan claramente la polaridad activopasiva, pero los modelos retraído, dependiente, independiente, y ambivalente no representan un cruce claro de las polaridades yo-otros y placer-dolor (Widiger, 1999). La Tabla 2 indica cómo los ocho modelos de personalidad se relacionan con las tres polaridades.

Tabla 2 - Los 8 patrones básicos de personalidad con respecto a las tres polaridades (adaptado de Widiger, 1999)

\begin{tabular}{|c|c|c|c|c|c|c|c|}
\hline \multicolumn{4}{|c|}{ Activo } & \multicolumn{4}{|c|}{ Pasivo } \\
\hline \multicolumn{2}{|r|}{$\mathrm{Yo}$} & \multicolumn{2}{|c|}{ Otros } & \multicolumn{2}{|r|}{ Yo } & \multicolumn{2}{|c|}{ Otros } \\
\hline Dolor & Placer & Placer & Dolor & Dolor & Placer & Placer & Dolor \\
\hline \multirow[t]{2}{*}{$\begin{array}{l}\text { Activo - } \\
\text { Retraído } \\
\text { (evitativo) }\end{array}$} & $\begin{array}{l}\text { Activo - } \\
\text { Independiente } \\
\text { (antisocial) }\end{array}$ & $\begin{array}{l}\text { Activo - } \\
\text { Dependiente } \\
\text { (histriónico) }\end{array}$ & & \multirow[t]{2}{*}{$\begin{array}{l}\text { Pasivo - } \\
\text { Retraído } \\
\text { (esquizoide) }\end{array}$} & $\begin{array}{l}\text { Pasivo - } \\
\text { Independiente } \\
\text { (narcisista) }\end{array}$ & $\begin{array}{l}\text { Pasivo- } \\
\text { Dependiente } \\
\text { (dependiente) }\end{array}$ & \\
\hline & $\begin{array}{r}\text { Activo - } A \\
\text { (pasivo- }\end{array}$ & $\begin{array}{l}\text { mbivalente } \\
\text { gresivo) }\end{array}$ & & & $\begin{array}{r}\text { Pasivo - } \\
(\mathrm{com}\end{array}$ & $\begin{array}{l}\text { mbivalente } \\
\text { alsivo) }\end{array}$ & \\
\hline
\end{tabular}

Durante la década que siguió la publicación de su libro, Millon influyó en el desarrollo de un nuevo sistema de clasificación psiquiátrico en lo que fueron las bases de la tercera edición del Manual Diagnóstico y Estadístico de los Trastornos Mentales. Aunque había algunas diferencias entre las personalidades de Millon y las del DSM-III, las conceptualizaciones de los prototipos de personalidad en los dos sistemas eran compatibles. La creación de ejes múltiples para el DSM-III, con un eje diseñado para sostener la composición de la 
personalidad del individuo, era fiel a la división de Millon entre personalidad patológica y otros tipos de psicopatología (Choca, 1999).

Los esfuerzos continuados para mejorar la nosología psiquiátrica llevaron a una pronta revisión del DSM-III en 1987. En particular, se discutió la posibilidad de agregar dos trastornos de personalidad, el sádico y el masoquista. Estos trastornos fueron incluidos en el apéndice de categorías diagnósticas que requieren estudios ulteriores en el DSM-III-R (APA, 1988) aunque fueron excluidos luego en el DSM-IV (APA, 1995). Sin embargo, estas nuevas categorías diagnósticas fueron incorporadas por Millon en la revisión que llevó a la segunda versión del Inventario Clínico Multiaxial de Millon [MCMI] (Millon, 1999b) aparecida al mismo tiempo que el DSM-III-R. Millon quedó convencido de la utilidad de ambas y las mantuvo en su modelo hasta la actualidad.

Para acomodar los nuevos trastornos Millon agregó un elemento discordante (que puede concebirse como una disposición orientada al dolor) a la naturaleza y fuente del refuerzo. Las personalidades discordantes utilizan las circunstancias conseguir refuerzo positivo o evitar refuerzo negativo, o para sustituir el dolor por el placer (Millon, 1999b). Como con los otros factores, el elemento discordante tiene una variante pasiva (la personalidad masoquista o autoagresiva) y una variante activa (la personalidad sádica o agresiva). Además de los nuevos prototipos, Millon revisó algunas de sus descripciones para aumentar la compatibilidad entre sus tipos de personalidad y los del DSM-III-R y cambió el nombre de la personalidad cicloide a personalidad límite.

Para la preparación de la próxima revisión del DSM que llevó a la cuarta edición (APA, 1995), el grupo de trabajo para los trastornos de la personalidad evaluó la posibilidad de agregar un desorden depresivo de personalidad. Aunque el prototipo se descartó y aparece en el apéndice del DSM-IV como una entidad que necesita de estudios futuros, Millon tomó este concepto y agregó el prototipo a su lista en la tercera versión del MCMI aparecida en 1994 (Millon, 1997b).
Otro notable hito en su desarrollo teórico ocurrió cuando Millon reformuló su teoría de la personalidad y sus trastornos para tener en cuenta los conceptos de la evolución mediante una teoría más evolutiva, filogenética, del desarrollo humano (Millon, 1990). El nuevo modelo procede a una reevaluación de las características más profundas en las que se basa el funcionamiento humano. La atención pasa de la psicología a otras expresiones de la naturaleza, examinando principios universales (de la evolución) derivados de manifestaciones no psicológicas. Cada especie muestra aspectos comunes en su estilo adaptativo, pero existen diferencias de estilo y de éxito adaptativo entre los miembros frente a los diversos y cambiantes entornos que enfrentan. La personalidad sería el estilo distintivo de funcionamiento adaptativo que exhibe un organismo o especie frente a sus entornos habituales. Los trastornos de la personalidad serían estilos particulares de funcionamiento desadaptativo.

La ampliación del modelo para incluir las fases de evolución no requirió una revisión significativa de las tres polaridades originales. El modelo evolutivo postula cuatro polaridades fundamentales de desarrollo, si bien los prototipos de personalidad se siguen explicando a partir de las tres primeras: (a) objetivos de existencia (polaridad placer-dolor), como se llega a ser - apertura (búsqueda de experiencias de recompensa) y preservación (evitación del peligro y la amenaza) de la vida; (b) modos de adaptación (polaridad activo-pasivo), como se sigue siendo - acomodación ecológica (atenerse a la realidad favorable) y modificación ecológica (dominio del propio entorno); (c) estrategias de replicación (polaridad yo-otros), como se transmite la existencia - individuación reproductora (consecución del sí mismo - Individualismo) y crianza reproductora (amor constructivo hacia los demás - protección); (d) procesos de abstracción, capacidad de simbolizar el mundo interno/externo - polaridad pensamiento sentimiento. La Tabla 3 muestra la clasificación de Millon de los trastornos de personalidad del DSM-III-R desde la perspectiva del modelo evolutivo (Millon, 1990).

Tabla 3 - Representación de los trastornos de personalidad del DSM-III-R con respecto al Modelo Evolutivo

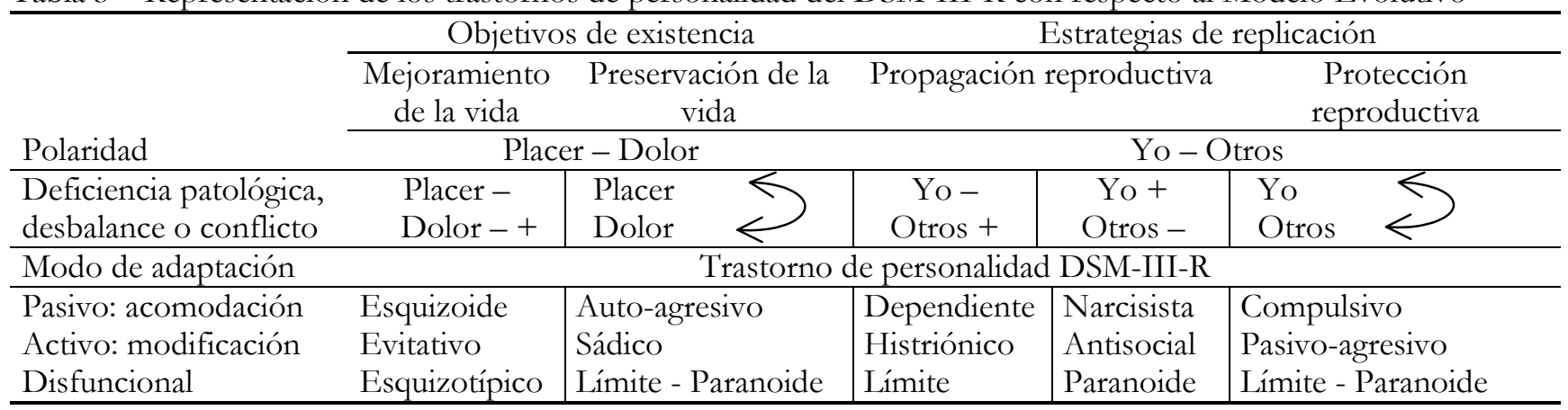


Los prototipos básicos de personalidad, coincidentes con los diferentes trastornos de personalidad que fueron apareciendo en las distintas versiones del DSM, fueron dando lugar a numerosas variaciones, resultantes de la investigación empírica y la experiencia clínica, que combinan aspectos de más de un trastorno básico (Millon \& Davis, 1998; Millon \& Davis, 2000; Choca \& Van Denburg, 1998). Así, por ejemplo, Millon concibió cuatro variaciones del trastorno evitativo de la personalidad que combinan, junto al patrón básico, rasgos pasivo-agresivos, paranoides, dependientes y depresivos, respectivamente (Millon \& Davis, 1998). Choca \& Van Denburg (1998), por su parte, ofrecen descripciones clínicas que combinan rasgos de tres patrones de personalidad diferentes. Por ejemplo, el perfil histriónico se combina con rasgos dependientes y negativistas, narcisistas y antisociales, narcisistas y negativistas, $y$ antisociales y negativistas.
Estas variaciones permiten al clínico una mayor discriminación entre pacientes diagnosticados (de acuerdo a los criterios del DSM) con un mismo trastorno.

Usando los tres juegos de polaridades, Millon pudo caracterizar cada uno de los prototipos de personalidad básicos. Un prototipo de personalidad puede ser fuerte, débil, o neutro en cualquier elemento particular de cualquiera de las polaridades. Ser fuerte en un elemento particular de una polaridad no implica un buen atributo, simplemente indica una tendencia hacia algún estilo de conducta. El resultado, para cada uno de los trastornos incluidos en la versión final del modelo (Millon \& Davis, 1998) se grafica en la Tabla 4. La tabla resulta bastante compleja aunque Millon simplificó esa complejidad dando énfasis a la polaridad de mayor deficiencia y agrupando los prototipos de personalidad como se muestra en la Tabla 5 (Choca, 1999).

Tabla 4 - Caracterización de los prototipos de Millon en término a las polaridades (adaptado de Choca, 1999)

\begin{tabular}{|c|c|c|c|c|c|c|}
\hline \multirow[t]{2}{*}{ Polaridades } & \multicolumn{2}{|c|}{ Objetivos de supervivencia } & \multicolumn{2}{|c|}{ Modos de adaptación } & \multicolumn{2}{|c|}{ Estrategia de replicación } \\
\hline & Placer & Dolor & Pasivo & Activo & Yo & Otros \\
\hline Esquizoide & $\mathrm{V}$ & $\mathrm{V}$ & 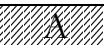 & V & & $\mathrm{V}$ \\
\hline Evitativo & $\mathrm{V}$ & W & $\mathrm{V}$ & 玨 & & \\
\hline Depresivo & $\mathrm{V}$ & $x$ & 留 & & & \\
\hline Dependiente & & & $x$ & $\mathrm{~V}$ & V & $x$ \\
\hline Histriónico & & & $\mathrm{V}$ & 垦 & $\mathrm{V}$ & 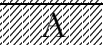 \\
\hline Narcisista & & & $x$ & $\mathrm{~V}$ & $x$ & $\mathrm{~V}$ \\
\hline Antisocial & & V & $\mathrm{V}$ & 紧 & & $\mathrm{V}$ \\
\hline Sádico & & $\mathrm{V}$ & $\mathrm{V}$ & - & & $\mathrm{V}$ \\
\hline Compulsivo & V & & 傮 & $\mathrm{V}$ & V & \\
\hline Negativista & $\mathrm{V}$ & & & 垡 & & $\mathrm{V}$ \\
\hline Autoagresivo & $\mathrm{V}$ & 1 & $x$ & & V & \\
\hline
\end{tabular}

Nota: El cuadro muestra la debilidad con una flecha hacia abajo y la fuerza con una flecha hacia arriba. Los cuadros grises muestran un punto neutro en la polaridad.

Tabla 5 - Agrupamiento actual de los prototipos de personalidad de Millon (adaptado de Millon \& Davis, 1998)

\begin{tabular}{cc}
\hline Personalidades con dificultades para el placer & Esquizoide \\
& Evitativo \\
Depresivo
\end{tabular}

Personalidades con problemas interpersonales Histriónico

Dependiente

Narcisista

Antisocial

Personalidades con conflictos intrapsíquicos

Sádico

Compulsivo

Negativista

Masoquista

Esquizotípico

Personalidades con déficit estructurales

Límite

Paranoide

Descompensado 
En defensa de su sistema de clasificación, Millon rechaza el agrupamiento del DSM-IV (APA, 1995). Sostiene que agrupar los trastornos por características descriptivas ("Los sujetos con estos trastornos suelen parecer...", expresa el DSM-IV) no tiene ninguna significación pronostica, ni significado etiológico, ni lógica alguna en términos de un modelo teórico (Millon \& Davis, 1998)

Estos han sido los principales aportes de Millon desde 1976 a la actualidad en lo que respecta a la teoría y a la clasificación de los trastornos de la personalidad. La teoría fue ganando en complejidad, lo que, al decir de Choca (1999) quizá sea el destino de cualquier teoría psicológica a medida que madura en su esfuerzo por reflejar las intrincadas complejidades de la naturaleza humana. Sin embargo, el modelo ha mantenido los lineamientos esbozados hace más de tres décadas. Otro tanto puede decirse de la clasificación, que se ha ido enriqueciendo a partir de las categorías básicas de 1976 con los distintos aportes surgidos de la investigación clínica y que fueran reflejados en algún momento en algunas de las versiones del DSM. Estos dos aspectos, teoría y clasificación, continúan siendo los pilares básicos de la propuesta de Millon para el estudio de la personalidad y su patología. Los otros dos componentes, la evaluación y el tratamiento, se enlazan con los anteriores en un intento de brindar medidas operacionales de los constructos teóricos y pautas para las intervenciones terapéuticas de los trastornos de la personalidad. Ambos componentes serán revisados a continuación.

\section{Evaluación e intervenciones terapéuticas}

Una vez sentados los lineamientos de su modelo, Millon se dedico a desarrollar diferentes instrumentos para operacionalizar sus constructos teóricos. En 1977 aparece la primera versión del primero de esos instrumentos: el Inventario Clínico Multiaxial de Millon (MCMI) (Strack, 1999), diseñado para evaluar trastornos de la personalidad. Con posterioridad, Millon elaboró otros inventarios de personalidad y clínicos, siempre con el propósito de proporcionar medidas operacionales de su modelo teórico. Se han publicado numerosos estudios usando estos inventarios para proveer evidencia empírica acerca de la validez del modelo. La gama de inventarios es amplia e incluye diferentes pruebas orientadas, por ejemplo, a la personalidad patológica (MCMI) (Millon, 1999b; Millon, 1997b), a los estilos de personalidad (Inventario Millon de Estilos de Personalidad - MIPS) (Millon, 1997a), a los adolescentes, evaluando tanto personalidad normal (Millon Adolescent Personality Inventory, MAPI) como patológica (Millon Adolescent Clinical Inventory, MACI), o a la salud (Millon Behavioral Health Inventory, MBHI) (Strack, 1999).

El más tradicional y más difundido de los instrumentos de evaluación de Millon es el MCMI que cuenta a la actualidad con tres versiones (Millon, 1999b; Millon, 1997b). Las revisiones (en 1987 y en 1994) fueron realizadas para adaptar el instrumento tanto a los cambios sufridos por la teoría cuanto a las modificaciones realizadas en la sección de trastornos de la personalidad en las distintas versiones del DSM.

Según Widiger (1999), el MCMI-III es quizás el instrumento más apropiado con el que evaluar empíricamente el modelo teórico de funcionamiento de la personalidad de Millon, como el instrumento que más directamente se relaciona con la evaluación del foco central de la teoría de los trastornos de la personalidad. También el MCMI-III podría ser el inventario de autoinforme favorito entre los clínicos para la evaluación de los trastornos de personalidad del DSM-IV (Widiger, 1999). El MCMI-II también resulta un fiel reflejo de la conceptualización teórica de Millon (Choca y otros, 1996). Un instrumento diagnóstico resulta más útil cuando está relacionado con una teoría comprensiva, como es el caso de esta prueba (Millon, 1992). Según Piotrowski (1997), una revisión de estudios sobre el tema indica que el MCMI ocupa un lugar preponderante entre los tests más frecuentemente usados en la práctica clínica, siendo solo superado por el MMPI en el área de la evaluación objetiva de la personalidad. Otro tanto sucede en la investigación, donde el MCMI resulta el tercer test más usado; sólo el MMPI-2 y el Rorschach han producido más investigaciones (Craig, 1999b).

Existe una controversia en torno a la capacidad del MCMI-III para medir los constructos de la teoría. Esta controversia se basa en que la mayoría de sus ítems fueron reescritos para representar los criterios diagnósticos del DSM-IV en lugar de los constructos teóricos yo-otros, placer-dolor, y activo-pasivo, propios de la teoría (Widiger, 1999). En tal caso, podría sostenerse que esta mayor adecuación con los criterios diagnósticos de la nosología oficial se contrapone, en cierto punto, con la evaluación del modelo teórico ya que no existe una coincidencia total entre lo que propone la teoría y lo que propone el DSM-IV. Así, es difícil determinar si cada escala del MCMI-III evalúa el modelo teórico o los criterios diagnósticos del DSM-IV para el trastorno correspondiente. Sobre el MCMI-II, instrumento ampliamente utilizado para evaluación clínica, no existe tal controversia, lo que, en principio, habilitaría a continuar con su utilización.

Otra discusión presente en la literatura actual sobre el MCMI está dirigida a los resultados que brinda 
la prueba. Algunos autores sostienen que las escalas básicas, o algunas de ellas, son más bien medidas de estilos de personalidad antes que de trastornos. Así, desde la perspectiva de Choca y sus colaboradores (Choca \& Van Denburg, 1998; Choca y otros, 1992), las ocho escalas básicas de personalidad originales miden estilos más que trastornos de la personalidad. Estos autores consideran que las puntuaciones altas de esas escalas básicas deben considerarse como una medida de las asunciones básicas de la persona, de sus actitudes más destacadas y su forma característica de interactuar, sin dar por sentado que cualquier elevación es señal de patología. Millon difiere con esta interpretación de los resultados del MCMI y sigue sosteniendo que las puntuaciones elevadas deben considerarse como indicador de la existencia de trastornos de la personalidad (Choca \& Van Denburg, 1998). En el mismo sentido, Craig (1999a) señala que si bien el MCMI es principalmente un instrumento para la evaluación diagnóstica de los trastornos de la personalidad, existen tres escalas (la histriónica, la narcisista y la compulsiva) que merecen especial atención. Según este autor, la mayor parte de los datos existentes sugieren que las escalas histriónica y compulsiva son medidas de estilos de personalidad y no de trastornos, mientras que la escala narcisista puede medir estilo o trastorno de la personalidad. Craig (1999a) no concuerda con la postura de Choca y Van Denburg (1998) según la cual todas las escalas básicas son medidas de estilo y no de trastornos de la personalidad, agregando que esta visión no es por lo general la más aceptada.

Más allá de las controversias citadas en los párrafos anteriores, el MCMI es un instrumento ampliamente usado tanto en investigación como en la práctica clínica. En sus tres versiones, ha generado más de 500 artículos y 6 libros, ha sido traducido a varios idiomas y está siendo usado en investigaciones transculturales (Craig, 1999a; Craig, 1999b). Gracias a este cúmulo de evidencia empírica, se cuenta en la actualidad con un bagaje de conocimientos substancial para tomar decisiones clínicas basadas en los resultados de esta prueba (Craig, 1999b). Si bien originalmente el instrumento fue diseñado para usar con población clínica, se han realizado algunas investigaciones con población no clínica (Craig, 1999b). En nuestro contexto, se ha utilizado el MCMI-II para evaluar personalidad en pacientes con enfermedades cardiovasculares internados en unidad coronaria (Sanchez, 2003; Urquijo, Sanchez, Monssón \& Heredia, 2001) y en alumnos ingresantes a la carrera de Psicología en una Universidad nacional (Sans, Calzetti y Morales, 2000).

El MCMI en sus versiones II y III contiene cinco tipos de escalas: escalas de validez y de tendencia de respuesta, escalas de trastornos de personalidad de gravedad leve, escalas de trastornos de personalidad más patológica, escalas de síndromes clínicos de gravedad moderada, y escalas de síndromes clínicos de gravedad acentuada. En total son 22 escalas clínicas (24 en el MCMI-III) y 4 escalas de validez y de tendencia de respuestas medidas a través de 175 ítems dicotómicos.

Además del acercamiento a la personalidad patológica, Millon, como se dijo anteriormente, concibe el término personalidad para designar el estilo más o menos distintivo de funcionamiento adaptativo que un miembro determinado de una especie muestra al relacionarse con su gama típica de ambientes. Concebida de este modo, la personalidad normal reflejaría los modos específicos de adaptación de un miembro de una especie que son eficaces en ambientes previsibles; los trastornos de personalidad representarían diferentes estilos de funcionamiento mal adaptados atribuibles a deficiencias, desequilibrios o conflictos en la capacidad de un miembro para relacionarse con los ambientes con que se enfrenta.

El instrumento para evaluar la personalidad normal es el Inventario Millon de Estilos de Persona-lidad [MIPS] (Millon, 1997a). Diseñado para utilizar con adultos normales, evalúa dimensiones de rasgos y estilos interpersonales característicos que existen en la población normal. La nueva perspectiva se desarrolló sin referencia a trastornos pero tomó prestados muchos conceptos del modelo original de personalidad.

El MIPS presenta una serie de ventajas sobre otras pruebas de personalidad, compartidas en cierta medida con lo que se destaca en el manual del MCMIII. En primer lugar, consta sólo de 180 ítems de respuesta verdadero/falso (permiten evaluar 24 escalas diferentes), un número lo suficientemente pequeño para que pueda usarse en diferentes contextos y no cansar demasiado a los sujetos, y lo suficientemente grande para permitir la evaluación de una gama amplia de comportamientos clínicamente relevantes. En segundo lugar, el instrumento está vinculado a una teoría clínica importante que le sirve de sustento. Una teoría bien elaborada, sostiene Millon (1997a), aporta más sencillez y claridad que la información dispersa y no integrada.

Para desarrollar el instrumento, Millon consideró el universo de rasgos y estilos interpersonales que existen en la población normal. El Inventario viene con tres sets de variables de personalidad que definen y miden esos rasgos y estilos; además, el MIPS contiene 3 indicadores de validez. Los tres conjuntos de variables son descritos a continuación.

Metas motivacionales. Representan los tres ejes básicos en forma evolutiva y evalúan la orientación de la persona a obtener refuerzo del medio. La polaridad placer-dolor se llamó apertura - preservación; activo - 
pasivo fue modificación - acomodación; y yo - otros individualismo - protección.

Modos cognitivos. Implican la fuente individual primaria para obtener información y la manera en que ésta es procesada. Las fuentes de información preferidas pueden ser uno mismo (introversión) o los otros (extroversión) y guiarse por la observación (sensación) o por la abstracción (intuición). El procesamiento puede basarse esencialmente en el intelecto (reflexión) o en los sentimientos (afectividad). Finalmente, puede existir la tendencia a asimilar la información a los contenidos previos (sistematización) o a generar conocimiento nuevo (innovación).

Conductas interpersonales. Describen diez estilos o conductas interpersonales; evalúan el estilo de relación con los demás. Ocho son esencialmente las mismas normales de 1976 y se relacionan con los trastornos de personalidad (véase Tabla 1). Dos adicionales, sometimiento y control, hipotéticamente se relacionan con los trastornos agresivo y auto-agresivo.

La evaluación de la personalidad, tanto cuando existe un trastorno como cuando no, resulta de utilidad para el trabajo clínico. Sin embargo, para que exista tal utilidad los instrumentos deben ser adecuados para los constructos que supuestamente miden. Los distintos inventarios de personalidad de Millon resultan buenas medidas operacionales de la teoría, aunque las investigaciones para dar cuenta de su validez deben continuarse.

El último aspecto que hace al planteamiento de Millon es el de las intervenciones psicoterapéuticas. Para Millon, la psicoterapia es la derivación lógica de una ciencia psicológica madura. La propuesta clínica del autor es la de una psicoterapia integradora, la cual resulta especialmente adecuada para el tratamiento de los trastornos de la personalidad (Millon, Everly \& Davis, 1995). En los últimos años, Millon ha trabajado en este último paso de su teoría, y ahora el modelo permite extrapolar objetivos terapéuticos del tratamiento a partir de los postulados de la teoría, la clasificación y la evaluación. La psicoterapia integradora es concebida como una configuración de estrategias y tácticas de las cuales se selecciona cada técnica de intervención no sólo por su eficacia en la resolución de ciertos síntomas patológicos concretos, sino también por su contribución a la constelación global de procedimientos terapéuticos de los cuales no es más que una parte (Millon \& Davis, 1998).

A partir del modelo teórico se deducen las implicaciones terapéuticas apuntando a los disbalances que pueden presentar las diferentes polaridades. En los trastornos de la personalidad, las polaridades pueden estar desbalanceadas de diferentes maneras describiendo la patología característica de cada trastorno específico (Dorr, 1999). El objetivo de cualquier tratamiento, entonces, es lograr cierto balance entre las polaridades. Por ejemplo, las personalidades con dificultad para el placer (esquizoide, evitativa y depresiva) se caracterizan por tener bajo el polo del placer en la polaridad placer-dolor (véase Tabla 4), así la estrategia general para estos pacientes sería que aumentaran su capacidad de experimentar placer en las experiencias de su vida.

Los últimos trabajos de Millon pasan por el desarrollo de un nuevo modelo de terapia integrativa, la psicosinergía, que resume sus esfuerzos de más de 30 años (Millon, 1999a; Millon, 2000). Cabe destacar que la integración que propone Millon no es inherente al tratamiento en sí, sino que la integración natural está en la persona (Millon \& Davis, 1998). Las personas, dice Millon, son el único sistema orgánicamente integrado en el área psicológica, creado inherentemente desde el nacimiento como entidades naturales, en lugar de gestalts derivadas de la experiencia. Psicosinergía (Millon 1999a; Millon, 2000) es el término que define la naturaleza única de la persona, la cual es en ocasiones fragmentada por razones pragmáticas. De esta manera, Millon recupera como sujeto principal del tratamiento psicológico a la persona más que a la patología.

Con la consideración de las intervenciones terapéuticas se cierra el círculo de los cuatro componentes básicos que propone Millon para una ciencia clínica comprensiva de la personalidad. Dichos componentes han sido sucintamente presentados hasta aquí en lo que respecta a sus aspectos centrales tal como han sido desarrollados por Millon y sus colaboradores desde 1976 hasta la actualidad.

\section{Conclusiones}

En sus escritos, Millon suele recurrir a Kurt Lewin quien escribió que "no hay nada más práctico que una buena teoría". De esta manera, Millon está expresando que el desarrollo consistente de una teoría es el requisito necesario para poder entender a la personalidad. A partir de allí, se podrá postular un sistema clasificatorio, diseñar instrumentos de evaluación y proponer una línea de tratamiento terapéutico. Así, como sostiene Millon (y como se ha mencionado reiteradamente en este trabajo), teoría, clasificación, evaluación y intervención, son los cuatro pilares que hacen a una ciencia clínica verdaderamente madura. Los esfuerzos de Millon, en todos estos años, han sido dirigidos en esa dirección y sus resultados han sido presentados aquí a modo introductorio. Cualquiera de los tópicos revisados amerita un mayor detenimiento y profundización para una cabal comprensión de los mismos.

La teoría ha ganado en complejidad y alcance pero manteniendo los lineamientos iniciales de 1976. 
Estos cambios fueron provocados por la necesidad de adecuar el marco teórico a los nuevos hallazgos surgidos de la investigación y de la práctica clínica. Tanto el modelo original de aprendizaje biosocial de 1976, cuanto la revisión evolucionista de 1990, o los sucesivos ajustes realizados en estas más de tres décadas de trabajo, dan cuenta del esfuerzo realizado y de la complejidad del objeto de estudio.

La clasificación se ha enriquecido incluyendo nuevos prototipos de personalidad, los cuales si bien estuvieron en algún momento dentro de la nosología oficial de la American Psychiatric Association (en las distintas versiones del DSM) posteriormente fueron abandonados o enviados al apartado de categorías diagnósticas que requieren estudios ulteriores. Millon se mostró más consecuente al respecto, adoptando las nuevas categorías e incluyéndolas en su modelo, lo que da por resultado una clasificación más fecunda y representativa de la problemática de la personalidad. Del mismo modo, esta inclusión enriquece el modelo teórico en un movimiento de realimentación continua entre teoría y clasificación.

La evaluación es el punto de enlace entre los conceptos teóricos y la realidad. Esto es así ya que mediante los instrumentos diseñados para evaluar personalidad (tanto normal como patológica) debe poder llegarse a un diagnóstico certero de los sujetos evaluados. Sí la teoría es correcta al momento de determinar los diferentes estilos y trastornos de la personalidad existentes en la población, los instrumentos diseñados a partir de ella deben ser capaces de determinar cual es estilo o trastorno de cada sujeto evaluado. Los inventarios de evaluación de Millon, en especial los destinados a población adulta (el MCMI y el MIPS), son los instrumentos que, dentro del modelo, fueron desarrollados para tales fines. Entonces, en principio, tanto el MCMI como el MIPS resultan adecuados para establecer que tipo de personalidad presenta toda persona que es diagnosticada con ellos. Sin embargo, tal adecuación no es algo dado de antemano sino que debe establecerse mediante pruebas empíricas. Hasta el momento, se ha visto que si bien tales instrumentos resultan de utilidad para evaluar los conceptos teóricos de Millon, queda margen para ciertas dudas. Es la investigación el único camino para despejar tales dudas. Los resultados de tales trabajo servirán no sólo para poder optimizar los instrumentos de evaluación sino también para revisar aspectos teóricos, de clasificación y de intervención, dado el complejo entramado que entre existe entre los mismos en el modelo de la personalidad de Millon.

La psicosinergía, el modelo terapéutico que propone Millon, es el punto final de este recorrido. Así, el modelo no solamente abarca a la personalidad patológica, a su clasificación y evaluación, sino también a las técnicas apropiadas para su tratamiento. $\mathrm{Y}$ si la base del modelo son las tres polaridades básicas revisadas en este trabajo, y la personalidad patológica implica un disbalance en esas polaridades, el tratamiento, entonces, tendrá como objetivo reducir esa disparidad. Este es el punto, tal vez, donde las opciones posibles excedan al modelo. Esto es así ya que existen diversas escuelas de psicoterapia que postulan como tratar a una persona con un trastorno de la personalidad. Sin embargo, esta diversidad de enfoques psicoterapéuticos no va en contra de los lineamientos de Millon. Por el contrario, los aspectos teóricos, clasificatorios y de evaluación, pueden enriquecer la práctica clínica más allá de los distintos abordajes.

El modelo de Millon se ha enriquecido enormemente en las últimas tres décadas lo que implica a su vez una mayor complejidad que atenta contra las explicaciones sencillas. Seguramente, tal complejidad se relaciona con la del objeto de estudio: la personalidad. Es la personalidad, en primera instancia, lo que debe estudiarse, lo que debe conocerse, como un paso previo necesario antes del conocimiento de su patología. Es la personalidad, ese constructo teórico que utilizamos para representar nuestra capacidad de percibir y enfrentar el mundo físico, psicológico y social, lo que se trata de entender. Y es a partir de propuestas teóricas fuertes, con amplio respaldo clínico y científico, por donde se encontrará el camino. En este trabajo se ha intentado mostrar una de esas propuestas, una que por su robustez y persistencia a lo largo del tiempo ha despertado el interés de clínicos e investigadores de diversas latitudes, y que mantiene su actualidad más de treinta años después de haber sido planteada.

\section{Referencias}

American Psychiatric Association (1968). DSM-II Diagnostic and statistical manual of mental disorders. Washington, DC: Mental Hospitals Service.

American Psychiatric Association (1988). DSM-III-R. Manual diagnóstico y estadístico de los trastornos mentales. Barcelona: Masson.

American Psychiatric Association (1995). DSM-IV Manual diagnóstico y estadístico de los trastornos mentales. Barcelona: Masson.

Choca, J. Evolution of Millon's Personality Prototypes (1999). Journal of Personality Assessment, 72(3), 353-364.

Choca, J. \& Van Denburg E. (1998). Guía para la interpretación del MCMI. Barcelona: Paidós.

Choca, J., Shanley, L., Van Denburg, E., Agresti, A., Mouton A. \& Uskokovic, L. (1992). Personality 
disorder or personality style: That is the question. Journal of Counseling \& Development, 70(3), 429-431.

Choca, J., Retzlaff, P., Strack, S., Mouton, A. e col. (1996). Factorial elements in Millon's personality theory. Journal of Personality Disorders, 10(4), 377-383.

Craig, R. (1999a). Overview and current status of the Millon Clinical Multiaxial Inventory. Journal of Personality Assessment, 72(3), 390-406.

Craig, R. (1999b). Testimony based on the Millon Clinical Multiaxial Inventory: Review, commentary, and guidelines. Journal of Personality Assessment, 72(2), 290-304.

Davis, R. (1999). Millon: Essentials of his science, theory, classification, assessment, and therapy. Journal of Personality Assessment, 72(3), 330-352.

Dorr, D. (1999). Approaching psychotherapy of the personality disorders from the Millon Perspective. Journal of Personality Assessment, 72(3), 407-425.

Millon, T. (1976). Psicopatología Moderna. Enfoque biosocial de los aprendizajes erróneos y de los disfuncionalismos. Barcelona: Salvat.

Millon, T. (1990). Toward a new personology: An evolutionary model. New York: Wiley.

Millon, T. (1992). Millon Clinical Multiaxial Inventory: I \& II. Journal of Counseling and Development, 70(3), 421-426.

Millon, T. (1997a). Inventario Millon de estilos de personalidad [MIPS]. Buenos Aires: Paidós.

Millon, T. (1997b). Millon Clinical Multiaxial Inventory III (MCMI III). 2nd ed. Minneapolis, MN: National Computers Systems.

Millon, T. (1999a). Reflection on Psychosynergy: A model for integrating science, theory, classification, assessment, and therapy. Journal of Personality Assessmen, 72(3), 437-456.

Millon, T. (1999b). Inventario clínico multiaxial de Millon-II [MCMI-II]. Manual. Madrid: TEA Publicaciones de Psicología Aplicada.

Millon, T. (2000). Toward a new model of integrative psychotherapy: Psychosynergy. Journal of Psychotherapy
Integration, 10(1), 37-53.

Millon, T. (2002). Assessment is not enough: The SPA should participate in constructing a comprehensive clinical science of personality. Journal of Personality Assessment, 78(2), 209-218.

Millon, T. \& Everly, G. (1994). La personalidad y sus trastornos. Barcelona: Martínez Roca.

Millon, T. \& Davis, R. (1998). Trastornos de la personalidad. Más allá del DSM-IV. Barcelona: Masson.

Millon, T. \& Davis, R. (2000). Personality disorders in modern life. New York: Wiley.

Millon, T., Everly, G. \& Davis, R. (1995). ¿Cómo puede facilitarse la integración de la psicoterapia mediante el conocimiento de la psicopatología? Clínica y Salud, 6(2), 109-129.

Piotrowski, C. (1997). Use of the Millon Clinical Multiaxial Inventory in clinical practice. Perceptual and Motor Skills, 84(3), 1185-1186.

Sanchez, R. (2003). Personalidad en pacientes con enfermedades cardiovasculares. Estudio a partir de la teoría de Millon. I Congreso Marplatense de Psicología, Mar del Plata, Argentina.

Sans, M., Calzetti, N. \& Morales, C. (2000). Perfil psicológico de los estudiantes que ingresan en la carrera de Psicología de la Universidad Nacional de San Luis. Idea, 14(30), 55-65.

Strack, S. (1999). Millon's Normal Personality Styles and Dimensions. Journal of Personality Assessment, 72(3), 426-436.

Urquijo, S., Sanchez, R., Monssón, N. \& Heredia, L (2001). Relaciones entre trastornos de la personalidad y enfermedades cardiovasculares. Psico-USF, 6(2), 75-84.

Widiger, T. (1999). Millon's Dimensional Polarities. Journal of Personality Assessment, 72(3), 365-389.

Recebido em abril de 2003 Reformulado em outubro de 2003 Aprovado em dezembro de 2003

Sobre o autor:

Roberto Oscar Sánchez é licenciado em Psicologia, com pós-graduação em Formação e Treinamento em Psicoterapia pela Fundación Aiglé, bolsista em pesquisa e professor da Facultad de Psicología da Universidad Nacional de Mar del Plata, Argentina. 
